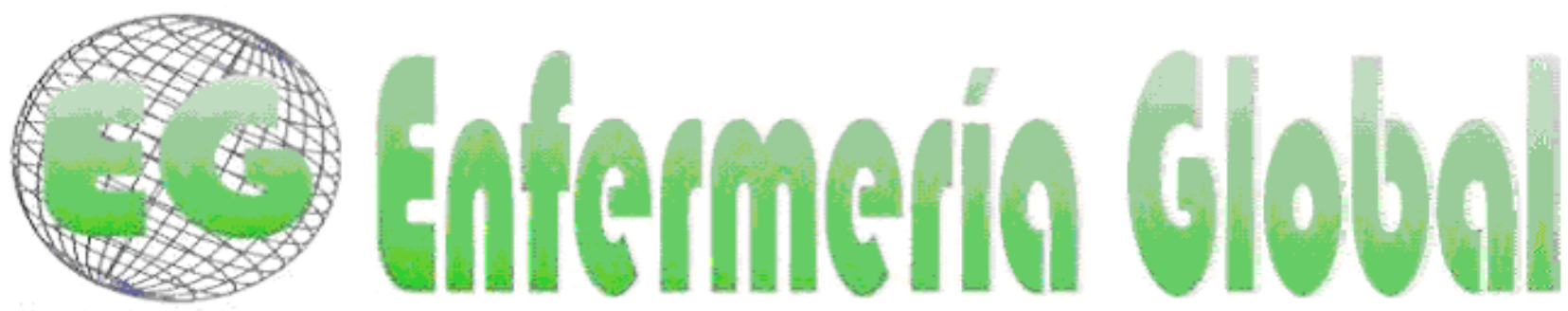

ISSN 1695-6141 Revista electrónica trimestral de Enfermería

$\mathrm{N}^{\circ} 23$

www.um.es/egloball

Julio 2011

ADMINISTRACIÓN - GESTIÓN - CALIDAD

\title{
Evaluación de la calidad de vida de pacientes de insuficiencia renal crónica en diálisis renal
}

Avaliação da qualidade de vida de portadores de insuficiência renal crônica em diálise renal.

\author{
${ }^{*}$ Cunha Franco, L., *Teles Zatta,L., ${ }^{* *}$ Vasconcelos, P., ${ }^{* * *}$ Alves Barbosa, M., \\ ${ }^{* * * *}$ Santana dos Santos, JR., ${ }^{* * * *}$ De Oliveira Rosa, LF. \\ "Enfermera. " Estudiante de Enfermería. ${ }^{* * *}$ Profesora Doctora de la Facultad de Enfermería. **** Médico. \\ Universidad Federal de Goiás. Brasil.
}

(Trabajo patrocinado por el CNPq).

Palabras clave: Calidad de vida; Diálisis renal; Estilo de vida; Insuficiencia renal crónica.

Palavras-chave: Qualidade de vida; Diálise renal; Estilo de vida; Insuficiência renal crônica.

Keywords: Quality of life; Renal dialysis; Life style; Renal insufficiency chronic.

\section{RESUMEN}

Objetivo: Evaluar la calidad de vida de las personas con Insuficiencia Renal Crónica (IRC) en hemodiálisis, a través de KDQOL TM-SF.

Métodos: Estudio descriptivo, analítico y cuantitativo, utilizando como instrumento para recolectar datos el KDQOL- SF TM, en una muestra de 32 pacientes con insuficiencia renal crónica. Las respuestas a las preguntas del cuestionario se distribuyeron en una escala dicotómica y en escalas tipo Likert, con puntuación que va desde diez hasta tres.

Resultados: Las puntuaciones más altas fueron en los campos: estímulo del equipo de hemodiálisis $(98,44)$, función sexual $(94,14)$ y síntomas y problemas $(76,04)$. Los menores encontrados: sobrecarga de la enfermedad renal $(12.34)$, función física $(23,44)$ y apoyo social $(30,21)$. Conclusión: Los aspectos principales que afectan a la calidad de vida de estas personas hacen reflexionar sobre un cuidado más planificado e integral que será prestado por el equipo de salud, médica, lo que permitirá vivir con más calidad.

\section{RESUMO}

Objetivo: Avaliar a qualidade vida de pessoas com Insuficiência Renal Crônica (IRC) em tratamento hemodialítico, através do KDQOL-SF ${ }^{\mathrm{TM}}$.

Métodos: Estudo descritivo-analítico, quantitativo, utilizando como instrumento de coleta de dados o KDQOL- SF TM, numa amostra de 32 pacientes com IRC. As respostas para as questões do questionário foram distribuídas em uma escala dicotômica e em escalas tipo Likert, com número de pontos variando entre dez e três. 
Resultados: Os maiores escores foram nos domínios: estímulo da equipe de hemodiálise $(98,44)$, função sexual $(94,14)$ e sintomas e problemas $(76,04)$. Os menores corresponderam: sobrecarga da doença renal $(12,34)$, função física $(23,44)$ e suporte social $(30,21)$.

Conclusão: Os aspectos que mais interferem na qualidade de vida dessas pessoas permitem reflexões a respeito de um cuidado mais planejado e integral a ser prestado pela equipe de saúde, permitindo um viver com mais qualidade.

\section{ABSTRACT}

Aim:To evaluate the quality of life of people with Chronic Renal Failure (CRF) in hemodialysis treatment through the KDQOL-SF ${ }^{\mathrm{TM}}$.

Methods: Study descriptive - analytical, quantitative, with KDQOL-SF ${ }^{\mathrm{TM}}$, in 32 patients with CRF. The answers to the questions of the questionnaire were distributed in a dichotomous scale and Likert scales, with the number of points ranging from ten to three.

Results: The highest scores were the areas: the stimulation of the hemodialysis team $(98,44)$, sexual function (94.14) and symptoms and problems (76.04). The lowest were: burden of renal disease (12.34), physical function (23.44) and social support (30.21).

Conclusion: The aspects that most affect the quality of life of these people encourage reflection about better planning and integral care by the health team, in order to attain a life of higher quality.

\section{INTRODUCCIÓN}

El concepto de calidad de vida (CV) ha sido utilizado desde la definición de salud de la Organización Mundial de la Salud (OMS) como estado completo de bienestar físico, psíquico y social, y no solamente ausencia de dolencia y enfermedad ${ }^{1}$.

Por la variabilidad de las definiciones de calidad de vida era necesario llegar a un consenso sobre un concepto que abarcara la mayoría de las características de los individuos en diferentes lugares y situaciones. Para esto, la OMS reunió a representantes de varios países que conceptuaron la calidad de vida como "la percepción del individuo de su posición en la vida, en el contexto de la cultura y sistema de valores en los que vive y en relación con sus objetivos, expectativas, estándares y preocupaciones" 2:1405.

La preocupación internacional acerca de tener instumentos para evaluar y medir la calidad de vida hicieron que los investigadores y las instituciones elaboraran instrumentos con esa finalidad $^{3}$. Entre los instrumentos utilizados en la medición de la calidad de vida, encontramos el SF-36 (Medical Outcomes Study 36 - Item Short-Form Health Survey) y El KDQOL- SF ${ }^{T M}$ (Kidney Disease and Quality-of-Life Short-Form), que son más comúnmente utilizados en pacientes con insuficiencia renal crónica.

EI KDQOL- SF ${ }^{\mathrm{TM}}$ fue desarrollado en 1994 por el grupo Kidney Disease and Quality of Life (KDQOL) Grupo de Trabajo / RAND patrocinado por la Universidad de Arizona (EE.UU.), por la necesidad de instrumentos psicométricos para evaluar la calidad de vida relacionada con la salud, específicamente la enfermedad renal ${ }^{4}$.

El apoyo social ofrecido al paciente renal crónica y cómo se percibe es de suma importancia para el desarrollo de nuevas estrategias para ayudar en la preservación de la calidad de vida, aliviar el sufrimiento ante la enfermedad que tanto debilita y convierte en dependiente ${ }^{5}$.

Los avances tecnológicos y terapéuticos em El área de diálisis han contribuido al aumento de la supervivencia de la enfermedad renal, pero sin permitir el retorno a la vida em relación a los aspectos cualitativos ${ }^{6}$. Debido al aumento de la supervivencia de estos pacientes, se 
ha dado mayor énfasis a las complicaciones que pueden disminuir la calidad de vida y aumentan la mortalidad. ${ }^{7}$

Actualmente en Brasil, hay 70.872 pacientes en tratamiento renal sustitutivo, de acuerdo con el Censo de la Sociedad Brasileña de Nefrología de 2006, con 64.306 pacientes en hemodiálisis y diálisis peritoneal en 6.566, y 95.02\% de los pacientes tienen el SUS (Sistema Único de Salud) como pagador del convenio ${ }^{8}$.

Teniendo en cuenta los problemas anteriores, surge la siguiente pregunta - ¿cómo ser paciente con insuficiencia renal crónica y hemodiálisis puede interferir en la calidad de vida del individuo?

Esta investigación fue diseñada teniendo en cuenta la importancia y la contribución de los estudios sobre CV en la vida cotidiana de las personas con IRC, y los pocos estudios que abordan este tema.

Medir la calidad de vida del paciente de enfermedad renal crónica es muy importante porque revela el impacto de la enfermedad en las actividades diarias, el afrontamiento y la aceptación de la cronicidad de la dolencia para un tratamiento eficaz

La escasa literatura científica que abordase la CV de los pacientes con IRC en tratamiento de hemodiálisis justifica el desarrollo de la investigación y podría allanar el camino para la consideración de la calidad de vida en la supervivencia de la enfermedad renal crónica.

Así se tuvo como objetivo evaluar la calidad de vida de las personas con IRC en tratamiento de hemodiálisis a través del instrumento KDQOL-SF ${ }^{\mathrm{TM}}$.

\section{MATERIALES Y MÉTODOS}

Estudio descriptivo y analítico realizado en pacientes con insuficiencia renal crónica tratados en la unidad de hemodiálisis de un hospital universitario en Goiânia, Goiás, en 2007.

El estudio incluyó a pacientes con insuficiencia renal crónica que estaban en hemodiálisis y tenían más de veintiún años. Siendo excluidos del estudio los pacientes mayores o menores de veintiún años que habían interrumpido sus tratamientos.

Se abordaron pacientes con IRC que estaban en tratamiento de hemodiálisis en la unidad de hemodiálisis del área de estudio, un total de 44 personas, 32 de ellos aceptaron participar, 11 se negaron y 1 no participó debido a limitaciones físicas. Atendiendo a la Resolución $\mathrm{N} \cong 196 / 96$ de la investigación con seres humanos ${ }^{9}$ el proyecto fue presentado a la Comisión de Ética en Investigación Médica Humana y Animal del Hospital de lãs Clínicas de la Universidad Federal de Goiás.

La recolecta de datos se realizó durante la sesión de hemodiálisis, en un entorno predefinido con los administradores de la unidad, a través de entrevistas individuales utilizando el instrumento KDQOL-SF ${ }^{\mathrm{TM}}$, que evalúa el funcionamiento y el bienestar de los pacientes con enfermedad renal crónica.

EI KDQOL-SF ${ }^{T M}$ es un instrumento específico que evalúa la enfermedad renal crónica, que se aplica a los pacientes sometidos a algún tipo de programa de diálisis. Se trata de un 
instrumento auto-aplicable con 80 ítems, divididos en 19 escalas, que lleva alrededor de 16 minutos el ser contestado.

EI KDQOL incluye el MOS 36-Item Short Form Health Survey (SF-36) como una medida genérica y se complementa con escalas de varios ítems, dirigidas a las preocupaciones particulares de los pacientes con IRC. El instrumento está publicado en la U. S. Renal Data System - annual Data Report (1999) que realiza registros epidemiológicos anuales en pacientes renales crónicos.

Las respuestas a las preguntas del cuestionario se distribuyeron en una escala dicotómica com espuestas sí / no, y em otras escalas tipo Likert, con puntuación que va desde diez hasta tres. Estas últimas miden intensidad, capacidad, frecuencia y evaluación, en realción con las últimas cuatro semanas de vida de la persona ${ }^{10}$.

\section{RESULTADOS}

Los datos fueron introducidos y analizados en el Statistical Package for Social Sciences (SPSS). Para cada dimensión fueron calculados la media e intervalo de confianza, establecido en 95\%, $p<0,05$ (Tabla 1).

Tabla 1. Media de cada parámetro evaluado en relación al aspecto general y específico de la enfermedad renal. Goiânia 2007.

\begin{tabular}{|l|c|c|c|}
\hline \multicolumn{1}{|c|}{ DIMENSIONES } & MÉDIA & \multicolumn{2}{c|}{ IC (95\%) da média } \\
\hline \multicolumn{1}{|c|}{ Aspecto general } & & Inferior & Superior \\
\hline La función física & 40,47 & 29,54 & 51,39 \\
\hline Función física & 23,44 & 8,26 & 38,61 \\
\hline Dolor & 67,42 & 55,96 & 78,89 \\
\hline General de Salud & 33,37 & 27,29 & 39,45 \\
\hline Bienestar emocional & 62,00 & 53,49 & 70,51 \\
\hline Función emocional & 64,58 & 47,88 & 81,29 \\
\hline Función Social & 56,64 & 46,79 & 66,49 \\
\hline Energía y Fatiga & 54,38 & 45,50 & 63,25 \\
\hline $\begin{array}{l}\text { Los aspectos } \\
\text { específicos de la } \\
\text { enfermedad renal }\end{array}$ & & & \\
\hline $\begin{array}{l}\text { Los síntomas y los } \\
\text { problemas }\end{array}$ & & & \\
\hline Efecto de la enfermedad & 63,87 & 70,04 & 81,77 \\
\hline La carga de la enfermedad & 12,34 & 0,30 & 7,71 \\
\hline Profesional de papel & 69,95 & 63,72 & 76,19 \\
\hline La función cognitiva & 45,39 & 40,58 & 50,20 \\
\hline Interacción Social & 75,00 & 67,99 & 82,01 \\
\hline Función Sexual & 94,14 & 87,18 & 101,10 \\
\hline Del sueño & 48,41 & 43,04 & 53,78 \\
\hline Apoyo Social & 30,21 & 17,67 & 42,74 \\
\hline $\begin{array}{l}\text { Estímulo del equipo de } \\
\text { diálisis }\end{array}$ & 98,44 & 95,25 & 101,62 \\
\hline Satisfacción del paciente & 66,15 & 61,21 & 71,09 \\
\hline & & & \\
\hline & & & \\
\hline
\end{tabular}


Los resultados de las dimensiones están en las puntuaciones que van de cero a cien, siendo la puntuación más alta el mejor estado. Las puntuaciones KDQOL se debe convertir en valores padronizados por KDQOL Working Group

Las dimensiones con mayor puntuación fueron: estímulo del equipo de hemodiálisis $(98,44)$, función sexual $(94,14)$, síntomas y problemas $(76.04)$ e interacción social $(75,00)$.

Los puntajes más bajos correspondieron a las siguientes categorías: sobrecarga de la enfermedad renal (12.34), función física $(23,44)$, apoyo social $(30,21)$ y salud general $(33,37)$.

\section{DISCUSIÓN}

La dimensión que presentó la puntuación más alta fue estímulo del personal de diálisis, que revela la satisfacción del paciente con el tratamiento ofrecido por los profesionales. Se señaló que los estudios de Cordeiro ${ }^{10}$ y Martín, Sarró, Ferrer, Arenas, González y Gil ${ }^{11}$ corroboran esta dimensión. Debido a que los pacientes en hemodiálisis permanecieron mucho tiempo en el lugar de tratamiento, terminan formando lazos afectivos con el equipo. ${ }^{11}$

La dimensión la función sexual se puntuó $(94,14)$, revelando que pocos pacientes tenían alteración de la actividad sexual. Este resultado también se asemeja a Cordeiro ${ }^{10}$ y Martin Sarró, Ferrer, Arenas, González y Gil ${ }^{11}$. Sin embargo, Lemos y Santos ${ }^{12}$ estudiaron la disfunción sexual de 52 pacientes en hemodiálisis de sexo masculino, a través de entrevistas, niveles hormonales por radioinmunoanálisis y evaluaciones de las concentraciones plasmáticas de zinc por espectrofotometría de absorción atómica, procediendo también a una amplia revisión de la literatura sobre el tema. La disfunción sexual se observó en El $98 \%$ de los pacientes.

La disparidad entre los datos presentados muestran que las puntuaciones más altas pueden estar enmascarados por la dificultad de abordar la cuestión de la sexualidad, generando la no confianza en el resultado, ya que em el estudio de Lemos y Santos ${ }^{12}$ casi el $100 \%$ de los

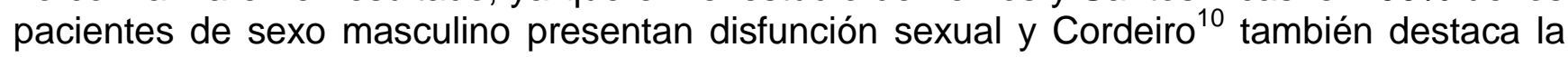
preocupación por las puntuaciones más altas en su estudio, pues encontró en la literatura que existe un deterioro grave de la función sexual en estos pacientes.

Otro alto puntaje fue síntomas y problemas (76.04) inherentes a la enfermedad renal. Esta dimensión evalúa el alcance del problema o síntoma que molesta al paciente en los últimos 30 días, incluye dolores musculares, en las articulaciones, espalda, pecho, cabeza, calambres durante la diálisis, piel seca, picazón en la piel, dificultad para respirar, debilidad o mareos, pérdida de apetito, cansancio, entumecimiento de manos o pies, náuseas y problemas de acceso vascular.

Otro aspecto que mostró resultados significativos fue la calidad de las interacciones sociales $(75,00)$. Los encuestados mostraron su disposición a socializar con sus familias haciendo hincapié en la importancia de esta interacción en la lucha contra la enfermedad renal.

De las categorías que obtuvieron puntuaciones más bajas, destaca la sobrecarga de la enfermedad renal (12.34). Esta dimensión evalúa los aspectos em que la enfermedad renal causa decepción o frustración y su influencia en la vida del paciente. 
Para los encuestados, tener una enfermedad crónica y ser sometidos a hemodiálisis continua, aparte de las complicaciones físicas y psicológicas causadas por esta enfermedad, ello causa un cambio enorme en su vida. Los pacientes dijeron que gastan gran parte de su tiempo con la enfermedad renal, por las varias sesiones que realizan durante la semana y la atención domiciliaria necesaria. Además de la sensación de decepción en el tratamiento de la enfermedad se sienten una carga para sus familias.

El segundo resultado más bajo fue la alteración de la función física $(23,44)$. Esta dimensión evalúa las limitaciones sobre el tipo y cantidad de trabajo realizado por el paciente, así como las actividades ordinarias y habituales. Todas las relacionadas con la apariencia física. En el estudio de Cordeiro ${ }^{10}$ esta dimensión corresponde a la puntuación más baja $(20,49)$, lo que significa que estas personas tienen gran dificultad en la realización de actividades sencillas de la vida diaria.

Los bajas puntuaciones relativas al apoyo social y salud general reflejan la insatisfacción de los pacientes sobre el tiempo y el apoyo recibido de amigos y familiares y una evaluación negativa de la situación actual y global de la salud.

Hoy en día, la tecnología y la preocupación en ofrecer un tratamiento eficaz, seguro y sin complicaciones, llevan tanto al enfermero como a los demás profesionales que prestan asistencia directa a la enfermedad renal crónica, a una atención poco específica de las particularidades de cada paciente.

\section{CONCLUSIÓN}

Las puntuaciones más altas mostraron que la mayoría de los encuestados estaban satisfechos con el apoyo recibido por el equipo de salud y la actividad social y sexual se vieron poco afectadas por la enfermedad. Las puntuaciones más bajas revelaron una alta sobrecarga de la enfermedad renal en la vida de los pacientes y grandes interferencias de la enfermedad en la función física, apoyo social y salud en general.

Debemos considerar que cada individuo tiene diferentes soportes físicos, mentales y sociales de afrontamiento y adaptación a la enfermedad renal y sus complicaciones. Por lo tanto, los resultados indican los aspectos que más afectan a la calidad de vida de estas personas, que son: sobrecarga de la enfermedad renal, función física, apoyo social y salud general, lo que lleva a la reflexión sobre un cuidado más planificado e integral proporcionado por el equipo de salud para estas personas.

Es comprensible entonces, que la promoción de la salud debe ser asumida como una responsabilidad de todos los sectores, ya que los gobiernos solos no pueden satisfacer las demandas sociales que determinan la salud. Un gran desafío hoy es el desarrollo de una sociedad más sana, fomentando el diseño de políticas públicas que promuevan la salud, la inversión en actividades de investigación y que se centren en la mejora de la calidad de vida de la población estimulando y fomentando la participación popular ${ }^{13}$.

\section{REFERENCIAS}

1- Spitzer WO. State of science 1986: quality of life and functional status as target variables for research. J Chorn Dis 1998; 40 (6): 465-71. 
2- The WHOQOL Group 1995. The World Health Organization quality of life assessment (WHOQOL): position paper from the World Health Organization. Soc sci and medicine 1995; 10 (1): 1403-09.

3- Laurenti R. A mensuração da qualidade de vida. Rev Assoc Med Bras (São Paulo) 2003; 49 (4): 361-62.

4- Duarte SP, Miayzaki MCOS, Ciconelli RM, Sesso R. Tradução e adaptação cultural do instrumento de avaliação de qualidade de vida para pacientes renais crônicos (KDQOLSF $^{\mathrm{TM}}$ ). Rev Assoc Med Bras [on line] (São Paulo). 2003; 49(4): 375-81. Disponível em: http://www.scielo.br/pdf/ramb/v49n4/18335.pdf. Acesso em 08 de abril de 2007.

5- Pedroso RS, Sbardelloto G. Qualidade de vida e suporte social em pacientes renais crônicos: revisão teórica. Psicópio: Revista Virtual de Psicologia Hospitalar e da Saúde. 2008; 4 (7): 32-35.

6- Madeira EQP, Lopes GS, Santos SFF. A investigação epidemiológica na prevenção da insuficiência renal terminal. Ênfase no estudo da agregação familiar. Medonline [on line] 1998; 1 (2). Disponível em: http://www.medonline.com.br/med ed/med2/epidemio.htm. Acesso em: 27 de maio de 2008.

7- Bastos Jr, Marco AV, Oliveira MMS, Castro SH de, Cunha EF, Moraes ERS, et al . Fatores de risco para o desenvolvimento de diabetes mellitus pós-transplante renal. Arq Bras Endocrinol Metab [on line] 2005; 49 (2): 271-77. Disponível em: http://www.scielo.br/scielo.php?script=sci arttext\&pid=S0004-27302005000200014\&lng. Acesso em 16 de agosto de 2008.

8- Sociedade Brasileira de Nefrologia Censo - janeiro/2006 [on line]. Disponível em: www.sbn.org.br/Censo/censo06htm. Acesso em 19 de setembro de 2008.

9- Ministério da Saúde; Comissão Nacional de Ética em Pesquisa, Conselho Nacional de Saúde. Resolução № 196/96 - Normas regulamentadoras de pesquisas envolvendo seres humanos. Brasília: Ministério da Saúde; 1996. Disponível em: www.conselho.saúde.gov.br/docs/reso196.doc.

10-Cordeiro JABL. Tratamento hemodialítico e qualidade de vida: avaliação do portador de insuficiência renal crônica. (Dissertação). Goiânia (GO): Faculdade de Enfermagem da Universidade Federal de Goiás; 2006.

11-Martín F, Sarró F, Ferrer R, Arenas D, Gozalez F, Gil T. Evaluaión de la calidad de vida en pacientes de una unidad de hemodiálisis con el cuestionario Kidney Disease Quality of Life - Short form (KDQOL - SF). Rev espan diálisis trasplante. 2004; 25 (1): 79-92.

12-Lemos VM, Santos OR. Disfunção sexual na insuficiência renal crônica sob hemodiálise regular /Sexual dysfunction in chronic kidney failure under maintenance hemodialysis. An Acad Nac Med. 1992; 152 (2): 8-13

13-Aerts D, Alves GG, La Salvia MW, Abegg C. Promoção de saúde: a convergência entre as propostas de vigilância da saúde e da escola cidadã. Cad saúde pública. 2004; 20 (4): 1020-28 